KCL-TH-08-02

IFT-P.004/2008

\title{
The cohomology of superspace, pure spinors and invariant integrals
}

\author{
N. Berkovits ${ }^{1}$ and P.S. Howe ${ }^{2}$ \\ 1 Instituto de Fisica Teorica, State University of São Paulo, Brasil \\ 2 Department of Mathematics, King's College, London, UK
}

\begin{abstract}
The superform construction of supersymmetric invariants, which consists of integrating the top component of a closed superform over spacetime, is reviewed. The cohomological methods necessary for the analysis of closed superforms are discussed and some further theoretical developments presented. The method is applied to higher-order corrections in heterotic string theory up to $\alpha^{\prime 3}$. Some partial results on $N=$ $2, d=10$ and $N=1, d=11$ are also given.
\end{abstract}




\section{Contents}

1 Introduction $\quad 1$

2 Invariant integrals $\quad 2$

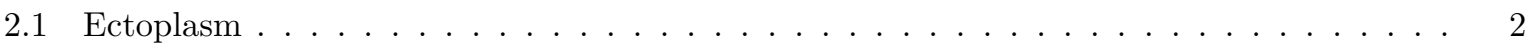

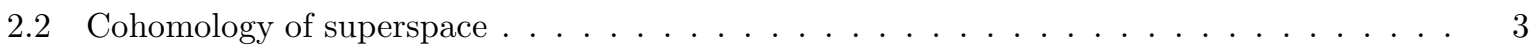

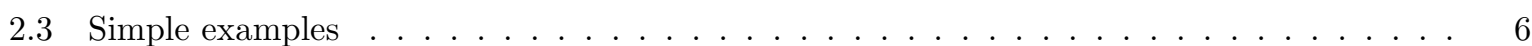

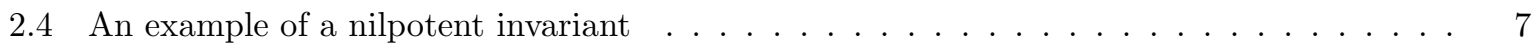

2.5 Chern-Simons invariants $\ldots \ldots \ldots \ldots \ldots \ldots \ldots \ldots \ldots$

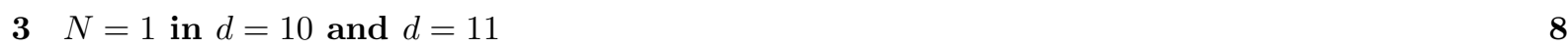

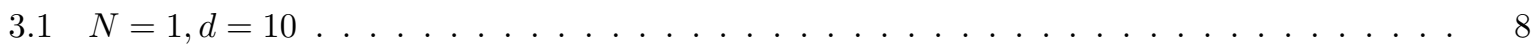

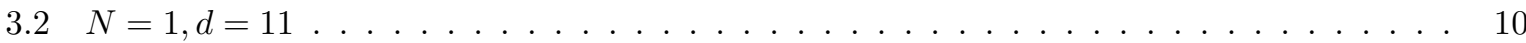

4 Heterotic invariants $\quad 10$

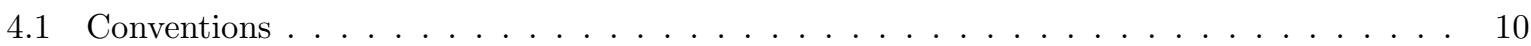

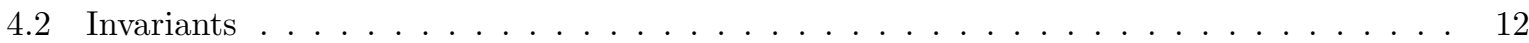

5 Invariants from superstring amplitudes $\quad 15$

5.1 Heterotic superstring amplitudes . . . . . . . . . . . . . . . . . . . . . . . 15

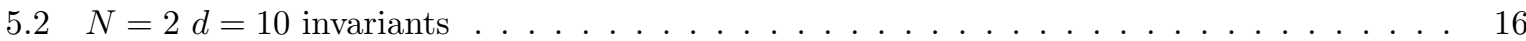

5.3 Proposal for $N=2$ invariants using doubled superspace $\ldots \ldots \ldots \ldots \ldots \ldots \ldots$

5.4 Remarks on $N=2, d=10$ cohomology $\ldots \ldots \ldots \ldots \ldots \ldots \ldots$

6 Discussion 20

\section{Introduction}

Pure spinors have turned out to be extremely useful in supersymmetric field theory and string theory. They were used in an attempt to solve the auxiliary field problem for $N=1, d=10$ super Yang-Mills (SYM) theory [1], but they made their first appearance in the modern sense in $[2,3]$ where it was shown how one could derive the equations of motion for $d=10, N=1$ SYM and supergravity, including the gauge sector Chern-Simons term, and for $d=11$ supergravity, from the postulate of pure spinor integrability. This amounts to assuming the existence of a BRST operator formed by contracting the pure spinor with a suitable fermionic derivative. In the case of supergravity one has to use either loop superspace or membrane superspace in order that the two- or three-form potentials in $d=10$ or $d=11$ supergravity can be reinterpreted as one-form gauge fields in the functional superspaces. Moreover, in $[4,5,6]$, a version of heterotic string theory was given with $N=2$ worldsheet supersymmetry in which pure spinor variables arise naturally. Somewhat later, the pure spinor variables were reinterpreted as ghosts [7] thereby giving rise to a version of superstring theory which can be quantised in a way which preserves spacetime supersymmetry manifestly. In a series of papers (see [8] for a review and references) it was shown how particles and various strings could be formulated in terms of pure spinors with the latter acting as ghosts for both kappa-symmetry and worldsheet reparametrisations. An attempt was also made to describe the membrane in a similar way but this is as yet not fully understood [9], while 
$d=11$ pure spinors have also been used to describe superparticles and topological M-theory [10, 11]. Supergravity constraints and pure spinors in $d=10$ and $d=11$ have recently been discussed from the perspective of free differential algebras $[12,13,14,15]$.

Pure spinors also arise naturally in the cohomology of superspace, called spinorial cohomology in [16]. Indeed, the spinorial cohomology groups for purely odd forms (i.e. all odd indices), turn out to be isomorphic to the pure spinor cohomology groups if we adopt the definition that a pure spinor $\lambda$ satisfies $\lambda \gamma_{a} \lambda=0$. This coincides with the usual definition in $d=10$ but not in $d=11$ where a pure spinor in Cartan's sense also obeys $\lambda \gamma_{a b} \lambda=0$. There are also spinorial cohomology groups with an additional vector index [16], relevant to the deformations of the dimension zero torsion, and groups for forms with mixed even and odd indices [17]. The main application of spinorial cohomology groups has been to the analysis of allowed deformations of theories with maximal supersymmetry for which it is believed that there are no off-shell versions $[18,19,16,20,17,21,22,23] .{ }^{1}$ The absence of auxiliary fields means that one cannot write down higher-order invariants straightforwardly; instead they have to be constructed using only the physical fields.

The construction of such invariants is the main topic of this paper. The idea is to combine the cohomological approach with the superform ("ectoplasm") method [25, 26, 27] of constructing invariant integrals in $d$-dimensional spacetime from closed $d$-forms in superspace. This was discussed for $d=11$ in [17] and the general theory has been described in a talk [28]; some results were also given in an earlier paper [29].

The organisation of the paper is as follows: in the next section we briefly review the superform method, we discuss superspace cohomology in general, including cohomology groups for odd forms taking their values in $\wedge{ }^{k} T_{0}$, where $T_{0}$ is the even tangent bundle, and we give some simple examples of invariants. In section 3 we discuss the cohomology groups for $N=1$ supersymmetry in $d=10$ and 11 and show how they can be used to construct invariants. In section 4 we apply the theory to the explicit construction of various invariants in the $N=1, d=10$ heterotic superstring effective action and show that most of them, up to order $\alpha^{\prime 3}$, can be completely determined in this manner. These $N=1, d=10$ invariants are also related to terms in the Type I superstring effective action by the usual heterotic-Type I duality symmetry. We then discuss in section 5 the relation of the invariants to pure spinor superstring scattering amplitudes, as well as possible generalisations to $d=11$ and $d=10, N=2$ supergravity theories.

\section{Invariant integrals}

\subsection{Ectoplasm}

In this subsection we shall briefly review the superform or ectoplasm method for constructing superinvariants $[25,26,27]$. This gives an elegant and practical way of computing superspace integrals if the dimension of spacetime is small enough and can also be useful for invariants which correspond to subsuperspace integrals. It is closely related to the notion of rheonomy in the group manifold approach to supersymmetry [30] and the generalized action principle [31].

Let $M$ be a supermanifold with $d$ even dimensions, and let $M_{0}$ denote its body. Let $s: M_{0} \rightarrow M$ be a section of the projection $\pi: M \rightarrow M_{0}$, and let $J_{d}$ be a closed $d$-form on $M$. We claim that the integral

$$
I:=\int_{M_{0}} s^{*} J_{d}
$$

is independent of the choice of section, provided that we are allowed to neglect surface terms. Since the vertical directions in $M$ correspond to the odd coordinates, it follows that the integral $I$ is invariant under supersymmetry. Note also that $I$ is unchanged if we replace $J_{d}$ by $J_{d}+d K_{d-1}$, so that we are really interested in the $d$ th de Rham cohomology group. Note, however, that we are only interested in forms that can be constructed from the physical fields of the theory under discussion, so that the relevant

\footnotetext{
${ }^{1}$ For a recent discussion of the non-maximal case see [24].
} 
cohomology group is $H_{d}^{d}($ phys), and this can be non-trivial even if the space itself is topologically trivial. Indeed, we shall assume that spacetime is topologically trivial throughout the paper.

We sketch a proof of this claim. Let $f_{t}$ be a one parameter family of diffeomorphisms of $M$ generated by a vector field $v$, define the family of sections $s_{t}$ by $s_{t}=f_{t} \circ s$ and set

$$
I_{t}=\int_{M_{0}} s_{t}^{*} J_{d}=\int_{M_{0}} s^{*} \circ f_{t}^{*} J_{d}
$$

Then

$$
\begin{aligned}
\left.\frac{d}{d t} I_{t}\right|_{t=0} & =\int_{M_{0}} s^{*} £_{v} J_{d} \\
& =\int_{M_{0}} s^{*}\left(d \iota_{v} J_{d}+\iota_{v} d J_{d}\right) \\
& =\int_{M_{0}} d\left(s^{*} \iota_{v} J_{d}\right) .
\end{aligned}
$$

Hence if we assume the fields in the integrand fall off fast enough at infinity, or if $M_{0}$ is compact, we see that the right-hand-side vanishes thus justifying the claim. In practice, it is usual to take $s=e$, the zero section, $e(x)=(x, 0)$. In this case we have

$$
I=\int d^{d} x \varepsilon^{m_{d} \ldots m_{1}} J_{m_{1} \ldots m_{d}}(x, 0) .
$$

The superspaces of interest to us have the property that their tangent bundles $T$ can be split invariantly into even and odd parts, $T=T_{0} \oplus T_{1}$, with corresponding local bases $E_{A}=\left(E_{a}, E_{\alpha}\right)$. If $v$ is an odd vector field, $v=v^{\alpha} E_{\alpha}$, we can identify the leading component of $v^{\alpha}$ with the parameter of local supersymmetry, and the above discussion shows that the integral is indeed supersymmetric.

Equation (2.5) allows one to compute a component invariant integral systematically even in a curved superspace. Letting $E_{M}{ }^{A}$ denote the supervielbein which relates coordinate bases to preferred bases, and identifying the spacetime vielbein and gravitino fields by $e_{m}{ }^{a}:=E_{m}{ }^{a} \mid$ and ${\psi_{m}}^{\alpha}:=E_{m}{ }^{\alpha} \mid$, where the bar denotes evaluation of a superfield at $\theta=0$, we have

$$
\begin{gathered}
I=\int d^{d} x \varepsilon^{m_{1} \ldots m_{d}} \\
\quad\left(e_{m_{d}}{ }^{a_{d}} \ldots e_{m_{1}}{ }^{a_{1}} J_{a_{1} \ldots a_{d}}+d e_{m_{d}}{ }^{a_{d}} \ldots e_{m_{2}}{ }^{a_{2}} \psi_{m_{1}}{ }^{\alpha_{1}} J_{\alpha_{1} a_{2} \ldots a_{d}}+\ldots\right. \\
\left.\ldots+\psi_{m_{d}}{ }^{\alpha_{d}} \ldots \psi_{m_{1}}{ }^{\alpha_{1}} J_{\alpha_{1} \ldots \alpha_{d}}\right)
\end{gathered}
$$

where each of the $J_{\mathrm{s}}$ is evaluated at $\theta=0$.

\subsection{Cohomology of superspace}

In order to tackle the cohomology question it is useful to introduce the notion of $(p, q)$ forms, forms with $p$ even and $q$ odd indices with respect to a preferred basis. If we let $\Omega_{p, q}$ denote the space of such forms ${ }^{2}$ we have

$$
\Omega_{p, q} \ni \omega=\frac{1}{p ! q !} E^{\beta_{q}} \ldots E^{\beta_{1}} E^{a_{p}} \ldots E^{a_{1}} \omega_{a_{1} \ldots a_{p} \beta_{1} \ldots \beta_{p}},
$$

\footnotetext{
${ }^{2}$ We use subscripts rather then the more usual superscripts for reasons which will become clear shortly.
} 
where $E^{a}\left(E^{\alpha}\right)$ are preferred even (odd) basis forms dual to the basis vector fields $E_{a}\left(E_{\alpha}\right)$ introduced above.

The exterior derivative splits into four parts,

$$
d=d_{0}+d_{1}+t_{0}+t_{1}
$$

with bi-degrees $(1,0),(0,1),(-1,2),(2,-1)$ respectively [32]. It is easiest to write these using covariant derivatives and the torsion. Thus $d_{0} \sim E^{a}\left(\nabla_{a}+T_{a} \cdot{ }\right)$ and $d_{1} \sim E^{\alpha}\left(\nabla_{\alpha}+T_{\alpha} \cdot{ }^{\cdot}\right)$ are even and odd derivatives while $t_{0}$ and $t_{1}$ are algebraic operations involving the dimension zero and three-halves components of the torsion tensor. The operation $t_{0}$ applied to a $(p, q)$-form consists of contracting one of the even indices with the upper index on the dimension zero torsion, $T_{\alpha \beta}{ }^{c}$, followed by symmetrisation over the $(q+2)$ odd indices.

The identity $d^{2}=0$ splits into various components,

$$
\begin{aligned}
t_{0}^{2} & =0 \\
d_{1} t_{0}+t_{0} d_{1} & =0 \\
d_{1}^{2}+d_{0} t_{0}+t_{0} d_{0} & =0
\end{aligned}
$$

together with some others which will not be needed in the following. The first of the above equations allows us to introduce the cohomology groups $H_{t}^{p, q}$, the space of $t_{0}$-closed $(p, q)$-forms modulo the $t_{0}$ exact ones [32]. The groups $H_{t}^{0, q}:=H_{t}^{q}$ can be thought of as (generalised) pure multi-spinors. When $N=1$ and the dimension-zero torsion takes its usual form, $T_{\alpha \beta}{ }^{c}=-i\left(\gamma^{c}\right)_{\alpha \beta}$, an element of this group can be represented by a multi-spinor with $q$ symmetrised indices which is gamma-traceless on each pair. Such an object is clearly equivalent to an $\omega$ of the form

$$
\omega=\lambda^{\alpha_{1}} \ldots \lambda^{\alpha_{q}} \omega_{\alpha_{1} \ldots \alpha_{q}}
$$

where $\lambda$ obeys the constraint

$$
\lambda^{\alpha}\left(\gamma^{a}\right)_{\alpha \beta} \lambda^{\beta}=0
$$

In $d=10$ such a spinor is a pure spinor in the sense of Cartan but this is not always the case.

We can also define $t_{0}$-cohomology groups for $(0, q)$-forms taking their values in $\wedge^{k} T_{0}$; these will turn out to be useful for finding the $H_{t}^{p, q}$ groups. To do this let us first define the space $\Omega_{p, q}^{k, l}$ consisting of $(p, q)$ forms taking their values in $\wedge^{k} T_{0} \otimes \wedge^{l} T_{1}$, i.e the space of $(p, q)$-forms which are also $(k, l)$-multivectors. The dimension-zero torsion can be made to act in two ways on this space: firstly, we define $t_{0}$ to act as before, i.e. ignoring the multivector indices, and secondly we define a new operation $t^{0}: \Omega_{p, q}^{k, l} \rightarrow \Omega_{p, q+1}^{k+1, l-1}$. In components these operations are given by

$$
\left(t_{0} \omega\right)_{a_{1} \ldots a_{p-1}, \alpha_{1} \ldots \alpha_{q+2}}^{b_{1} \ldots b_{k}, \beta_{1} \ldots \beta_{l}}=\frac{(q+1)(q+2)}{2} T_{\left(\alpha_{1} \alpha_{2}\right.}{ }^{c} \omega_{\left.\left|c a_{1} \ldots a_{p-1}\right|, \alpha_{3} \ldots \alpha_{q+2}\right)}^{b_{1} \ldots b_{k}, \beta_{1} \ldots \beta_{l}}
$$

and

$$
\left(t^{0} \omega\right)_{a_{1} \ldots a_{p}, \alpha_{1} \ldots \alpha_{q+1}}^{b_{1} \ldots b_{k+1}, \beta_{1} \ldots \beta_{l-1}}=(-1)^{p+q+1}(k+1)(q+1) \omega_{a_{1} \ldots a_{p},\left(\alpha_{1} \ldots \alpha_{q}\right.}^{\left[b_{1} \ldots b_{k},\left|\beta_{1} \ldots \beta_{l-1} \gamma\right|\right.} T_{\left.\alpha_{q+1}\right) \gamma}{ }^{\left.b_{k+1}\right]} .
$$

It is straightforward to show that $t:=t_{0}+t^{0}$ is nilpotent,

$$
t^{2}=0 \quad \Leftrightarrow \quad\left(t_{0}\right)^{2}=\left(t^{0}\right)^{2}=t_{0} t^{0}+t^{0} t_{0}=0
$$


The operation $t$ maps $\oplus \Omega_{p-r, q+r}^{k-r, l+r}$ to $\oplus \Omega_{p-r-1, q+r+2}^{k-r, l+r}$ where the sum is over all integers $r$. We shall be interested in the cohomology groups $\left(H_{t}\right)_{0, q}^{k, 0}:=H_{t}^{q}\left(\wedge^{k} T_{0}\right)$. Since elements of $\Omega_{0, q}^{k, 0}$ are annihilated by $t_{0}$ and $t^{0}$, this group is given by elements of this space modulo elements of the form $t_{0} \lambda+t^{0} \rho$ where $\lambda \in \Omega_{1, q-2}^{k, 0}$ and $\rho \in \Omega_{0, q-1}^{k-1,1}$.

The groups $H_{t}^{p, q}$ will form the starting point for the analysis of the cohomology groups we are interested in. To go further we shall define the spinorial cohomology groups $H_{s}^{p, q}$. To do this we first define an odd derivative $d_{s}$ which acts on elements of $H_{t}^{p, q}$. We set

$$
d_{s}[\omega]:=\left[d_{1} \omega\right]
$$

where the square brackets denote equivalence classes in $H_{t}$ [17]. This definition makes sense because $t_{0}$ anticommutes with $d_{1}$. This means that the right-hand side is unchanged if $\omega \rightarrow \omega+t_{0} \rho$, for some $\rho \in \Omega_{p+1, q-2}$, and also that $t_{0} d_{1} \omega=0$ because $t_{0} \omega=0$. It is now easy to show that $d_{s}^{2}=0$. We have

$$
\begin{aligned}
d_{s}^{2}[\omega] & =d_{s}\left[d_{1} \omega\right] \\
& =\left[d_{1}^{2} \omega\right] \\
& =\left[-\left(t_{0} d_{0}+d_{0} t_{0}\right) \omega\right] \\
& =0,
\end{aligned}
$$

where we have used (2.9). Given that $d_{s}^{2}=0$ we can define the cohomology groups $H_{s}^{p, q}$ in the obvious way: $H_{s}^{p, q}:=H_{d_{s}}\left(H_{t}^{p, q}\right)$. The groups $H_{s}^{0, q}$ are isomorphic to the pure spinor cohomology groups $H_{Q}^{q}$. The latter are defined by acting on multi-pure spinors $\omega$ of the type given in (2.11) by $Q=\lambda^{\alpha} \nabla_{\alpha}$. This operator squares to zero in a supergravity background if the latter obeys pure spinor integrability.

We note in passing that one can also define spinorial cohomology groups for $(0, q)$-forms taking their values in $\wedge{ }^{k} T_{0}$. Let $h \in \Omega_{0, q}^{k, o}=\Omega_{0, q}\left(\wedge^{k} T_{0}\right)$. We can define an odd exterior derivative on such objects as follows:

$$
\left(d_{1} h\right) \cdot \omega=d_{1}(h \cdot \omega)+(-1)^{q+1} h \cdot d_{1} \omega
$$

where $\omega \in \Omega_{k, 0}$ and where the dot denotes contraction on all the even indices. Explicitly,

$$
\begin{aligned}
\left(d_{1} h\right)_{\alpha_{1} \ldots \alpha_{q+1}}^{a_{1} \ldots a_{k}}= & (q+1) \nabla_{\left(\alpha_{1}\right.} h_{\left.\alpha_{2} \ldots \alpha_{q+1}\right)}+\frac{q(q+1)}{2} T_{\left(\alpha_{1} \alpha_{2}\right.}{ }^{\gamma} h_{\left.|\gamma| \alpha_{3} \ldots \alpha_{q+1}\right)}^{a_{1} \ldots a_{k}} \\
& +(-1)^{q+1} k(q+1) h_{\left(\alpha_{1} \ldots \alpha_{q}|b|\right.}^{\left[a_{1} \ldots a_{k-1} \mid \alpha_{\left.\alpha_{q+1}\right) b}{ }^{\left.a_{k}\right]} .\right.}
\end{aligned}
$$

A straightforward computation shows, provided that the dimension zero torsion is covariantly constant, that

$$
d_{1}^{2} h=t_{0} \lambda+t^{0} \rho
$$

for some (computable) $\lambda \in \Omega_{1, q}^{k, 0}$ and $\rho \in \Omega_{0, q+1}^{k-1,1}$. We can therefore define $d_{s}[h]=\left[d_{1} h\right]$ and $H_{s}^{q}\left(\wedge^{k} T_{0}\right)=$ $H_{d_{s}}\left(H_{t}^{q}\left(\wedge^{k} T_{0}\right)\right)$.

Now suppose we want to compute the de Rham cohomology group $H_{d}^{n}$; we have to find an $n$-form $J_{n}$ satisfying $d J_{n}=0$, modulo shifts of the form $J_{n} \rightarrow J_{n}+d K_{n-1}$. The lowest-dimensional component of $J$ is $J_{0, n}$; it satisfies 


$$
t_{0} J_{0, n}=0
$$

trivially and is subject to the gauge transformation

$$
\delta J_{0, n}=t_{0} K_{1, n-2} .
$$

It is therefore given by an element of $H_{t}^{0, n}$. Now consider the $(0, n+1)$ component of $d J=0$,

$$
d_{1} J_{0, n}+t_{0} J_{1, n-1}=0 \text {. }
$$

This is equivalent to

$$
d_{s}\left[J_{0, n}\right]=0 .
$$

This pattern continues as the dimension is increased: the possible solutions to the de Rham cohomology problem are generated by elements of the spinorial cohomology groups $H_{s}^{p, q}$ where $p+q=n$. To find possible integral invariants using the superform method we therefore have to study the groups $H_{s}^{p, q} ; p+q=d$. However, it should be borne in mind that not every such group element will lead to an integral invariant in flat superspace because it could fail to contain $J_{d, 0}$ amongst its derived components. These are the invariants we are most interested in and will be the focus of the rest of the paper. The invariants which vanish in flat superspace will be referred to as nilpotent invariants.

\section{$2.3 \quad$ Simple examples}

A very easy example is given by $N=1, d=2$ superspace. The standard geometry is determined by conventional constraints. The non-zero components of the torsion tensor are

$$
\begin{aligned}
T_{\alpha \beta}{ }^{c} & =-i\left(\gamma^{c}\right)_{\alpha \beta} \\
T_{a \beta}{ }^{\gamma} & =\left(\gamma^{c}\right)_{\beta}^{\gamma} S \\
T_{a b}{ }^{\gamma} & =-i \varepsilon_{a b}\left(\gamma_{5}\right)^{\gamma \delta} \nabla_{\delta} S,
\end{aligned}
$$

where $S$ is a scalar superfield whose components are a dimension one auxiliary field, the gravitino field strength and the curvature scalar. The cohomology group $H_{t}^{0,2}$ consists of scalar functions which can be identified with possible superspace Lagrangians. Using the freedom to make $K$ transformations we can choose

$$
J_{0,2}=i \gamma_{0,2}^{\natural} J_{0},
$$

where $\gamma_{0,2}^{\natural}$ denotes " $\gamma_{5}$ " considered as a $(0,2)$-form. Similarly, we write

$$
\gamma_{p, 2}=\frac{1}{2 . p !} E^{a_{p}} \ldots E^{a_{1}} E^{\beta} E^{\alpha}\left(\gamma_{a_{1} \ldots a_{p}}\right)_{\alpha \beta} .
$$

It is easy to compute the components of $J_{2}$; they are

$$
\begin{aligned}
& J_{\alpha \beta}=i\left(\gamma_{5}\right)_{\alpha \beta} J_{0} \\
& J_{a \beta}=\left(\gamma_{5} \gamma_{a}\right)_{\alpha \beta} \nabla^{\beta} J_{0} \\
& J_{a b}=-\varepsilon_{a b}\left(i \nabla^{\alpha} \nabla_{\alpha} J_{o}+2 S J_{0}\right) .
\end{aligned}
$$


Given a particular $J_{0}$ we can compute its components and then find the spacetime action using (2.6). For example, the action for a spinning string may be obtained by taking

$$
J_{0}=\nabla_{\alpha} X \cdot \nabla^{\alpha} X
$$

where $X$ is the string field.

In $d=2$, for any $N$, the curvature two-form $R_{a b}$ is equal to $\varepsilon_{a b} F$, where $d F=0$. The supergravity action, which is purely topological as for $N=0$, is then given by setting $J_{2}=F$.

In $d=3$ the standard $N=1$ superspace constraints are also purely conventional, with $T_{\alpha \beta}{ }^{c}=-i\left(\gamma^{c}\right)_{\alpha \beta}$ again. In this case we need to look for closed three-forms. It is easy to see that $H_{t}^{0,3}=0$ and that $H_{t}^{1,2}$ is the space of scalar functions. We can choose $J_{1,2}$ to be

$$
J_{1,2}=i \gamma_{1,2} J_{0}
$$

where $J_{0}$ is the superspace Lagrangian. As in the $d=2$ case is it straightforward to compute the component action using (2.6).

A more complicated example is provided by $d=4, N=1$ supergeometry. The groups $H_{t}^{0,4}$ and $H_{t}^{1,3}$ are certainly not zero, but they cannot be used to generate Lagrangians forms which give rise to nonzero invariants in flat superspace. The simplest possibility arises at the next order and makes use of a non-vanishing element of $H_{t}^{2,2}$ [26]. In two-component notation we can take

$$
J_{a b \dot{\gamma} \dot{\delta}}:=J_{\alpha \dot{\alpha}, \beta \dot{\beta}, \dot{\gamma} \dot{\delta}}=\varepsilon_{\alpha \beta} \varepsilon_{(\dot{\alpha}|\dot{\gamma}|} \varepsilon_{\dot{\beta}) \dot{\delta}} J_{0}
$$

which will lead to a solution of $d J=0$ if $J_{0}$ is a chiral superfield, $\bar{\nabla}_{\dot{\alpha}} J_{0}=0$. This gives rise to a chiral invariant, i.e. an integral over chiral superspace of $J_{0}$. To get a full superspace integral one simply has to write $J_{0}$ in terms of two anti-chiral derivatives acting on some scalar superfield $S$.

The chiral example generalises straightforwardly to $d=4, N=2$ and indeed to higher $N$, although these are more complicated. There are other types of invariant in $N=2$ as can be seen from the superaction approach [33] or from harmonic superspace [34] (see [35] for a discussion of the relation between the two). In [36] some examples were derived from higher-rank closed forms in harmonic superspace from which one can obtain closed four-forms by integrating over the harmonic two-sphere.

\subsection{An example of a nilpotent invariant}

Consider on-shell $d=4, N=1$ supergravity in the absence of matter. The field strength superfield is a chiral dimension three-halves field $W_{\alpha \beta \gamma}$ obeying

$$
\nabla_{\alpha} W_{\beta \gamma \delta}=C_{\alpha \beta \gamma \delta}
$$

where $C_{\alpha \beta \gamma \delta}$ is the totally symmetric Weyl spinor. If we set

$$
J_{\alpha \beta \gamma \delta}=C_{\alpha \beta \gamma \delta}
$$

with all other components of $J$ with four spinor indices taken to vanish, then we claim that $d J=0$ if the other non-vanishing components of $J$ are

$$
\begin{aligned}
J_{a \beta \gamma \delta} & =\frac{1}{4} \nabla_{a} W_{\beta \gamma \delta} \\
J_{a b \gamma \delta} & =\varepsilon_{\dot{\alpha} \dot{\beta}}\left(\frac{1}{4} W_{(\alpha \beta}{ }^{\epsilon} W_{\gamma \delta) \epsilon}+\varepsilon_{(\alpha|\gamma|} \varepsilon_{\beta) \delta} W^{2}\right),
\end{aligned}
$$


where we have used the standard correspondence between a vector and a pair of spinor indices and where $W^{2}$ denotes the complete contraction of two $W \mathrm{~s}$. For example, the fact that $\nabla_{(\alpha} J_{\beta \gamma \delta \epsilon)}=0$ follows immediately from (2.21).

\subsection{Chern-Simons invariants}

The invariants that can be constructed using the superform method fall broadly into two classes: strict invariants, for which all the non-vanishing components of $J$ are tensorial, and the remainder, which can involve gauge potentials or perhaps explicit $\theta$ s. Many examples of invariants of the second type arise as Chern-Simons invariants, i.e. they include Chern-Simons terms together with, generically, many other tensorial terms which are required by supersymmetry. The general theory of such terms is as follows [37]: let $W$ be a closed $(d+1)$-form on a superspace $M$ whose body $M_{0}$ has dimension $d$, and suppose that $W$ is written explicitly as $d Z$ where $Z$ is a (local) $d$-form that involves gauge potentials; if $W$ can also be written as $d K$, where $K$ is a tensorial $d$-form, then

$$
J:=K-Z
$$

is a closed $d$-form which one can use to form invariants using the superform method. $Z$ obviously gives rise to the Chern-Simons or Wess-Zumino term while $K$ will give the rest of the bosonic terms which go with it. Since $W$ is a $(d+1)$-form it follows that it is exact in de Rham cohomology since the latter coincides with that of the body for a supermanifold. However, it might not be the case that it is exact for the right coefficients, which will be the space of physical fields in most examples, and therefore exactness, or Weil triviality [38], has to be checked explicitly in each case. A class of examples of this type is provided by Green-Schwarz actions for various branes [31, 37]; the superform method therefore provides a rather neat explanation of the relation between the kinetic and Wess-Zumino terms in such actions. Another example is the $R^{4}$ invariant in $d=11$ which includes the anomaly-cancelling Chern-Simons term for the fivebrane [17].

\section{$3 \quad N=1$ in $d=10$ and $d=11$}

In this section we shall study the theory of the possible invariants that can arise in supergravity backgrounds in $N=1, d=10$ and $d=11$. We shall make the analysis subject to the assumption that the $H_{t}$ cohomology groups are determined by the allowed $p$-branes. We do not have a proof of this statement but we know of no counterexample.

\section{1 $N=1, d=10$}

The allowed $p$ branes have $p=1$ or $p=5$. These couple to two- and six-form potentials respectively and there are therefore associated closed three- and seven-form field strengths. The dimension-zero components of these are proportional to $\gamma_{1,2}$ and $\gamma_{5,2}$ respectively, both of which are annihilated by $t_{0}$.

The group $H_{t}^{0, q}:=H_{t}^{q}$ is isomorphic to the space of totally symmetric, gamma-traceless $q$-spinors, i.e. the space of pure $q$-spinors. For $p=1$ it is easy to see that $H_{t}^{1,0}=0$ while $H_{t}^{1,1}$ is equal to the space of odd vector fields. An example of this occurs in on-shell $d=10$ super Maxwell theory. If $F$ denotes the field strength two-form, $F_{0,2}=0$, so that $t_{0} F_{1,1}=0$, and the solution to the latter equation being given

by an element of $H_{t}^{1,1}$; in fact, it is the physical fermion field. There are two contributions to $H_{t}^{1,2}$; the first is given by $(1,2)$-forms $\omega$ of the form

$$
\omega_{1,2}=\gamma_{1,2} f
$$


where $f$ is an arbitrary function. However, for $q>2$, there are no further non-trivial solutions that can be constructed using $\gamma_{1,2}$; this follows from the identity

$$
\left(\gamma_{a}\right)_{(\alpha \beta} \omega_{\left.\gamma_{1} \ldots \gamma_{r}\right)}=\left(\gamma^{b}\right)_{(\alpha \beta}\left(\gamma_{b a}\right)_{\gamma_{1}}{ }^{\delta} \omega_{\left.\gamma_{2} \ldots \gamma_{r}\right) \delta},
$$

where $r=q-2$. In other words, $t_{0}$-closed $(1, r+2)$-forms of the type $\gamma_{1,2} \omega_{0, r}$ are $t_{0}$-exact.

The remaining non-trivial possibilities are generated with the aid of $\gamma_{5,2}$. We find

$$
H_{t}^{p, q} \supseteq H_{t}^{q-2}\left(\Lambda^{5-p} T_{0}\right)
$$

provided that $p \leq 5$ and $q \geq 2$. To illustrate this consider a $t_{0}$-closed $(3, q+2)$-form $\omega$; if it is non-trivial it can be written

$$
\omega_{3, q+2}=\gamma_{5,2} \lambda_{0, q}^{2,0},
$$

where the notation indicates that two of the even indices of $\gamma_{5,2}$ are contracted with the even indices on $\lambda$. It is easy to see that changing $\lambda$ by $t_{0} \rho_{1, q-2}^{2,0}$ will lead to a $t_{0}$-closed change to $\omega$ and it is not difficult to see that the same will be true if we change $\lambda$ by $t^{0} \rho_{0, q-1}^{1,1}$. Hence the correct cohomology group is indeed $H_{t}^{q}\left(\wedge^{k} T_{0}\right)$ as claimed. If the brane assumption is correct, therefore, the non-vanishing $H_{t}^{p, q}$ cohomology groups for $N=1, d=10$ are

$$
\begin{aligned}
& H_{t}^{0, q}=H_{t}^{q} \\
& H_{t}^{1,1}=\Omega_{0,0}^{1,0} \\
& H_{t}^{1, q}=H_{t}^{q-2}\left(\Lambda^{4} T_{0}\right)+\delta_{q 2} \Omega_{0,0}^{0,0}, q \geq 2 \\
& H_{t}^{p, q}=H_{t}^{q-2}\left(\Lambda^{5-p} T_{0}\right), q \geq 2 ; p \in\{2,3,4,5\} .
\end{aligned}
$$

In order to form superinvariants the possible starting points for constructing closed ten-forms are given by the cohomology groups $H_{t}^{p, q}$ with $p+q=10$ and $p \leq 5$. We have $H_{t}^{0,10}=H_{t}^{10}$, the space of ten-fold pure spinors, while $H_{t}^{p, q} \cong H_{t}^{q-2}\left(\Lambda^{5-p} T_{0}\right)$ for $1 \leq p \leq 5$. However, it turns out that only $H_{t}^{5,5}$ can give rise to non-nilpotent integral invariants, because it is not possible to obtain non-zero $J_{10,0} \mathrm{~s}$ from any of the other possibilities. An element of $H_{t}^{5,5}$ can be written

$$
J_{5,5}=\gamma_{5,2} f_{0,3}
$$

where $f_{0,3}$ determines an element of $H_{t}^{3}$. It is easy to see that the lowest-order constraint on $J_{5,5}$ coming from $d J=0$ will be satisfied if

$$
d_{s}\left[f_{0,3}\right]=0,
$$

or, equivalently, $f$ is in third pure spinor $Q$-cohomology group. Starting from this, one can go on to find a solution for the rest of $J$. Note that if one were to be interested in nilpotent invariants starting from some other $J_{p, q}$, for example $J_{3,7}$, then one would have to make use of the generalised spinorial cohomology groups $H_{s}^{k}\left(\Lambda^{l} T_{0}\right)$.

In flat superspace the pure spinor cohomology groups were analysed previously in [39], where it was shown that the only possibilities arise for $0 \leq q \leq 3$ and that $H_{Q}^{3}$ corresponds to possible actions. This provides direct confirmation of the claim made above that there are no other possibilities. On the other hand, the superform method remains valid in the presence of a non-trivial supergravity background; if we can find elements of $H_{s}^{0,3}$ then we can systematically find complete invariants using the superform construction. 


\section{$3.2 \quad N=1, d=11$}

The situation in $d=11$ is slightly simpler from the cohomological point of view since the only scalar brane is the membrane. Thus the $H_{t}$ groups are generated by $\gamma_{2,2}$ which is $t_{0}$ closed, but not exact, by the membrane identity. The non-trivial $H_{t}$ groups are

$$
\begin{aligned}
& H_{t}^{0, q} \cong H_{t}^{q} \\
& H_{t}^{1, q} \cong H_{t}^{q-2}\left(T_{0}\right), \quad q \geq 2 \\
& H_{t}^{2, q} \cong H_{t}^{q-2}, \quad q \geq 2 .
\end{aligned}
$$

To find a closed 11-form we therefore need to consider $H_{t}^{11}, H_{t}^{8}\left(T_{0}\right)$ and $H_{t}^{7}$. The analysis of [9] suggests that it is the last of these groups which is important for non-nilpotent invariants. Thus we need to start with a $(2,9)$-form of the type

$$
J_{2,9}=\gamma_{2,2} f_{0,7}
$$

If $\left[f_{0,7}\right]$ satisfies

$$
d_{s}\left[f_{0,7}\right]=0
$$

this procedure will generate a closed 11-form and hence an invariant. Again, this analysis will be valid in the presence of a non-trivial supergravity background.

\section{Heterotic invariants}

\subsection{Conventions}

The on-shell constraints for $d=10, N=1$ supergravity were first written down in [40] and there have since been many reformulations. For our purposes it is most convenient to describe $d=10$ supergravity in terms of the partially on-shell $128+128$ multiplet [41]; it is dual to the supercurrent multiplet which is conformal in the sense that the energy-momentum tensor vanishes, but which has non-local aspects [42]. The SG multiplet consists of the graviton, the gravitino and the six-form potential $B_{6}$ with field strength $H_{7}=d B_{6}$; in practice we will use the dual of $H_{7,0}$ which we write as $G_{a b c}$. The partial on-shell nature is reflected in the constraints which hold for the scalar curvature and the double gamma-trace of the gravitino field strength $\Psi_{a b}$. There is a fully off-shell version of the supergravity theory [41], consisting of this multiplet together with two entire scalar superfields, but one of the latter has dimension -6 and can only be non-zero starting at order $\alpha^{\prime 3}{ }^{3}$ As we shall only be interested in the field equations up to order $\alpha^{\prime 2}$ it is therefore reasonable to take the supergravity constraints to be those of the $128+128$ multiplet $[43,44]$. We can study the equations of motion by introducing the two-form potential $B_{2}$ and its modified field strength $H_{3}$ satisfying $d H_{3}=\alpha^{\prime} X_{4}: \quad X_{4}:=\operatorname{Tr}\left(F^{2}-R^{2}\right)$ where $F$ is the SYM field strength tensor; this was done for the Chapline-Manton theory in $[45,46,47]$ and more generally in $[48,49,32,50,51,52]$ (see also $[53,54,55]$ for a different perspective, and $[56,57]$ for a dual approach). Note, however, that at order $\alpha^{\prime 3}$ the constraints must be changed because $H_{7}$ obeys the modified Bianchi identity, $d H_{7}=\alpha^{\prime 3} X_{8}$, where $X_{8}$ is an invariant eight-form constructed from $R, F$ whose form is determined by the anomalycancellation mechanism; indeed, it was noted that this has to be the case in [60]. Moreover, it is precisely at this order that the negative-dimension auxiliary field can first be non-zero and this also implies a modification of the dimension-zero torsion [59, 44].

\footnotetext{
${ }^{3}$ The second scalar superfield has dimension zero and contains the dilaton and dilatino.

${ }^{4} \mathrm{An}$ up-to-date summary and clarification can be found in [58].
} 
The torsion tensor is given by

$$
\begin{aligned}
T_{\alpha \beta}{ }^{c} & =-i\left(\gamma^{c}\right)_{\alpha \beta} \\
T_{\alpha \beta}{ }^{\gamma} & =T_{\alpha b}{ }^{c}=0 \\
T_{a b}{ }^{c} & =0 \\
T_{a \beta}{ }^{\gamma} & =\left(\gamma^{b c}\right)_{\beta}^{\gamma} G_{a b c}+\frac{1}{6}\left(\gamma_{a b c d}\right)_{\beta}^{\gamma} G^{b c d} \\
T_{a b}{ }^{\gamma} & =\Psi_{a b}{ }^{\gamma} .
\end{aligned}
$$

We can decompose the gravitino field-strength into irreducible, gamma-traceless components:

$$
\Psi_{a b}=\psi_{a b}+\gamma_{[a} \psi_{b]}+\gamma_{a b} \psi,
$$

and the constraint referred to above is simply that $\psi=0$. The components of the curvature tensor are given by

$$
\begin{aligned}
R_{\alpha \beta, c d} & =4 i\left(\left(\gamma^{e}\right)_{\alpha \beta} G_{c d e}+\frac{1}{6}\left(\gamma_{c d e f g}\right)_{\alpha \beta} G^{e f g}\right) \\
R_{\alpha b, c d} & =\frac{i}{2}\left(\gamma_{b} \Psi_{c d}-\gamma_{d} \psi_{b c}+\gamma_{c} \psi_{b d}\right)_{\alpha}
\end{aligned}
$$

while the leading component of $R_{a b, c d}$ is the spacetime curvature. The curvature scalar obeys the constraint

$$
R=12 G_{a b c} G^{a b c} .
$$

The non-zero components of $H_{7}$ are

$$
H_{a b c d e \alpha \beta}=-i\left(\gamma_{a b c d e}\right)_{\alpha \beta},
$$

or, more concisely, $H_{5,2}=-i \gamma_{5,2}$, and

$$
H_{a b c d e f g}=-2 \varepsilon_{a b c d e f g h i j} G^{h i j} .
$$

We shall only need the three-form to zeroth order in $\alpha^{\prime}$. Its non-zero components are

$$
\begin{aligned}
H_{\alpha \beta c} & =-i S\left(\gamma_{c}\right)_{\alpha \beta} \\
H_{a b \gamma} & =-\left(\gamma_{a b} \chi\right)_{\gamma}
\end{aligned}
$$

as well as $H_{a b c}$. The scalar field $S=\exp 2 \phi / 3$, where $\phi$ is the dilaton, and the dilatino is defined by $\chi_{\alpha}=D_{\alpha} S$. The $H_{3}$ Bianchi identity also implies that

$$
G_{a b c}=\frac{1}{12} \exp \left(-\frac{2 \phi}{3}\right) H_{a b c}
$$


and

$$
D_{\alpha} \chi_{\beta}=\frac{i}{2}\left(\gamma^{a}\right)_{\alpha \beta} D_{a} S-\frac{i}{36} H_{a b c} .
$$

The above equations are valid in what we shall refer to as the brane frame; the relation between the bosonic metrics in the two frames is

$$
g_{B}=\exp \left(-\frac{2 \phi}{3}\right) g_{S}
$$

At leading order the SYM field strength $F$ obeys the usual constraint that $F_{\alpha \beta}=0$; it follows that

$$
F_{\alpha b}=\left(\gamma_{b} \Lambda\right) \alpha,
$$

where $\Lambda^{\alpha}$ is the gaugino field. We then find that

$$
\begin{aligned}
D_{\alpha} \Lambda^{\beta} & =-\frac{i}{4}\left(\gamma^{a b}\right)_{\alpha}^{\beta} F_{a b} \\
D_{\alpha} F_{a b} & \left.=2\left(\gamma_{[a}\right) D_{b]} \Lambda\right)_{\alpha}-2\left(T_{[a} \gamma_{b]} \Lambda\right) \alpha
\end{aligned}
$$

where $T_{a}$ denotes the dimension-one torsion viewed as a matrix in spin space. In principle, there could be corrections to $F_{\alpha \beta}$ at order $\alpha^{\prime}$, but we shall not consider these here as it is probable that such corrections vanish. At order $\alpha^{\prime 2}$, however, there is the well-known correction which corresponds to the $F^{4}$ term in the Born-Infeld action $[61,62]$. In flat superspace it is proportional to

$$
\stackrel{(2)}{F}_{\alpha \beta}=\alpha^{\prime 2}\left(\gamma^{a b c d e}\right)_{\alpha \beta} \Lambda \gamma_{a b c} \Lambda F_{d e} .
$$

In a supergravity background this has to be modified slightly:

$$
\stackrel{(2)}{F}_{\alpha \beta}=\alpha^{\prime 2}\left(\gamma^{a b c d e}\right)_{\alpha \beta} \Lambda \gamma_{a b c} \Lambda\left(e^{2 \phi / 3} F_{d e}+\frac{i}{2} \chi \gamma_{d e} \Lambda\right),
$$

in the brane frame.

\subsection{Invariants}

Higher-order corrections to the heterotic string action have been studied for many years, see, for example, $[63,64,65,66]$. The simplest complete supersymmetric invariant that can be written down in $d=10$ is $[67]$

$$
I=\int d^{10} x d^{16} \theta E g(\phi)
$$

where $E$ is the superdeterminant of the supervielbein. Clearly supersymmetry does not completely fix this because it is an invariant for any function $g$. It will lead to an $R^{4}$ term in spacetime as long as the fourth derivative of $g$ is not zero. The heterotic tree-level $R^{4}$ term is of this type. In the ectoplasmic approach this invariant is generated by an $f_{0,3}$ which is schematically of the form $D^{11} g(\phi)$. It is interesting to note that the so-called non-minimal anomaly-free supergravity models have a non-vanishing $H_{0,3}$ which has a similar structure $[68,69]$. 
The remaining invariants we shall consider are of the Chern-Simons type and are completely fixed by supersymmetry. We shall not go into the full details here but we shall show that such invariants do indeed exist and identify the corresponding $J_{5,5} \mathrm{~s}$ in some cases. There are two possible closed eleven-forms:

$$
W^{(1)}:=\alpha^{\prime 3} H_{3} X_{8} \quad \text { and } \quad W^{(2)}=\alpha^{\prime} H_{7} X_{4} .
$$

In order to show that these define invariants of the Chern-Simons type we have to show that they can be written in the form $W^{(i)}=d K^{(i)}$ for some tensorial $K \mathrm{~s}$. Consider $W^{(1)}$ : since we are only working to order $\alpha^{\prime 3}$ we only need to know the field strengths to zeroth order. We know that $F_{0,2}=0$, but it can be shown that it is also possible to choose $R_{0,2}=0$ at this order on-shell [38]. This means that the lowest non-zero component of $X_{8}$ will be $X_{4,4}$, and therefore the lowest component of $W^{(1)}$ is $W_{5,6}^{(1)}$. The task is to show that this can be written as $t_{0} K_{6,4}^{(1)}$; if this is the case then the fact that $H_{t}^{p, q}=0$ for $p \geq 6$ indicates that there are no further obstructions to the existence of a suitable $K^{(1)}$. Since $X_{8}$ is closed it follows that $t_{0} X_{4,4}=0$ and so $X_{4,4}=\gamma_{5,2} X_{0,2}^{1,0}$. So

$$
\begin{aligned}
W_{5,6} & \sim \gamma_{1,2}\left(\gamma_{5,2} X_{0,2}^{1,0}\right) \\
& \sim \gamma_{5,2}\left(\gamma_{1,2} X_{0,2}^{1,0}\right) \\
& \sim t_{0}\left(\gamma_{5,2} X_{1,2}\right)
\end{aligned}
$$

where $X_{1,2}$ is the form obtained by lowering the upper even index on $X_{0,2}^{1,0}$ and where we have made use of $\gamma_{1,2} \gamma_{5,2}=0$. Thus the result is established; the invariant can be obtained from $J=K-Z$ by the general procedure. Since these terms include the anomaly-cancelling CS terms $Z$ it follows that they arise at one-loop in the heterotic string, but one can explicitly check that the correct factor of $e^{\phi}$ (i.e. no factor in the string frame) is present in the fourth-order field strength terms which arise from $K^{(1)}$.

Now let us consider $W^{(2)}$. Its lowest component is $W_{5,6}^{(2)} \sim \gamma_{5,2} X_{0,4}$. In this case we know from the BPT theorem [32] that $\operatorname{Tr}(R \wedge R)$ can be written as an exact form up to a four-form whose leading component is of type $(2,2)$, from which it follows that the geometrical part of $W^{(2)}$ is guaranteed to be of the required $d K^{(2)}$ form to the order we are considering. The same will be automatically true for the $\operatorname{Tr}(F \wedge F)$ term, up to order $\alpha^{\prime 2}$, provided that there is no order $\alpha^{\prime}$ correction to $F_{0,2}$, which we assume to be the case. We therefore conclude that supersymmetric invariants can indeed be constructed from $W^{(2)}$.

In the case of $W^{(2)}$ we can consider expanding the field strengths up to order $\alpha^{\prime 2}$, so that we can obtain invariants at first, second and third order in $\alpha^{\prime}$. For example, at $\alpha^{\prime 3}$ there will be terms involving $F^{4}$ coming from $\operatorname{Tr}(R \wedge R)$. These can arise as follows: since $R_{0,2} \sim G$, we find that $K_{6,4}^{(2)}$ has term from $\operatorname{Tr}(R \wedge R)$ of the form $G^{2}$. Now at order $\alpha^{\prime}$ it is easy to see that there is a contribution to $G$ of the form $e^{-2 \phi / 3} \Lambda^{2}$ and hence $K_{6,4}^{(2)}$ contains $e^{-4 \phi / 3} \Lambda^{4}$. Since we need four odd derivatives to arrive at $K_{10,0}$ we will therefore obtain an $F^{4}$ term in the spacetime invariant. It turns out that this term is at tree level in string theory; i.e. it has a factor of $e^{-2 \phi}$ in the string frame. As expected from the analysis of [63], this term is proportional to $\operatorname{Tr}\left(F^{2}\right) \operatorname{Tr}\left(F^{2}\right)$ since it comes from the square of the Yang-Mills scalar $G$. On the other hand, there is a second way of obtaining $F^{4}$ and that is from the $\alpha^{\prime 2}$ deformation of $F_{0,2}$. This term is proportional to $\operatorname{Tr}\left(F^{4}\right)$ and is actually a one-loop term and must therefore be partnered with the $B_{2} X_{8}$ in the effective string action. This is to be expected as it is easy to see in components that the equations of motion of the two-form and six-form theories with CS terms and modified field strengths are indeed equivalent.

For both of the CS invariants we have been discussing, the lowest component of $K$ is at least $K_{6,4}$, so that the lowest relevant component of $J$, namely $J_{5,5}=\gamma_{5,2} f_{0,3}$, actually comes from the CS term. We shall conclude this subsection by working out $f_{0,3}$ explicitly for the one-loop SYM $F^{4}$ term from both $W$ s. To make life easier we shall consider an abelian gauge field and a flat supergravity background. Consider first $H_{7} F^{2}$ : the CS potential $Z$ can be chosen to be $H_{7} Y_{3}$ where $Y$ is the CS three-form for $F^{2}$. Thus 


$$
J_{5,5}=\gamma_{5,2} Y_{0,3}
$$

and hence $f_{0,3}=Y_{0,3}$. Now we have to include the $\alpha^{\prime 2}$ corrections in $F^{2}$, so that

$$
Y_{\alpha \beta \gamma}=A_{(\alpha} \stackrel{(2)}{F}_{\beta \gamma)},
$$

and of course one takes the gamma-traceless part in the action. This expression agrees with the $f_{0,3}$ calculated explicitly from string amplitudes in the pure spinor approach as expected (see section 5).

On the other hand, in the $H_{3}$ version, we have $Z=H_{3} X_{4} Y_{3}$ in the abelian case with $X_{4}=F^{2}$. The lowest component of $Z$ is therefore $Z_{4,6} \sim H_{1,2} F_{1,1} F_{1,1} Y_{1,2}$. We first show that this is $t_{0}$ exact. We note that the only non-zero component of $H$ in flat space is $H_{1,2} \sim \gamma_{1,2}$ while the fact that $F_{0,2}=0$ implies that

$$
\begin{aligned}
Y_{0,3} & =0 \\
t_{0} Y_{1,2} & =0 \\
d_{1} Y_{1,2}+t_{0} Y_{2,1} & =0 .
\end{aligned}
$$

Let us define $M:=H_{3} F$; it is straightforward to show that its lowest component, $M_{2,3}$, can be written

$$
M_{2,3}=t_{0} N_{3,1},
$$

where $N_{a b c \delta} \sim\left(\gamma_{a b c} \Lambda\right)_{\delta}$. Using (4.20) and (4.21) we find

$$
Z_{4,6}=-t_{0}\left(N_{3,1} F_{1,1} Y_{1,2}\right)
$$

and so can be gauged away. At the next level

$$
Z_{5,5}=H_{1,2}\left(\left(F_{1,1}\right)^{2} Y_{2,1}+2 F_{1,1} F_{2,0} Y_{1,2}\right) .
$$

With a little algebra it is not difficult to see that

$$
Z_{5,5}+d_{1}\left(N_{3,1} F_{1,1} Y_{1,2}\right)=\left(M_{3,2}-d_{1} N_{3,1}\right)\left(F_{1,1}\right)^{2} A_{0,1}+t_{0} \text { - exact } .
$$

Since $d M=0$ it follows that $M_{3,2}-d_{1} N_{3,1}$ is $t_{0^{2}}$-closed. The exact part of this can be ignored in (4.24), and the non-trivial part is proportional to $\gamma_{5,2} F^{2,0}$ (where $F^{2,0}$ is $F_{2,0}$ with raised indices). The constant of proportionality cannot be zero since then $Z_{5,5}$ would also be trivial and the entire invariant would be a total derivative which it clearly is not. We also know that $F_{1,1}^{2} \sim \gamma_{5,2} L^{3,0}$, where $L^{a b c}=\Lambda \gamma^{a b c} \Lambda$. Hence, up to gauge terms

$$
Z_{5,5} \sim\left(\gamma_{5,2} F^{2,0}\right)\left(\gamma_{5,2} L^{3,0}\right)
$$

If we discard all the gauge terms the resulting $Z_{5,5}$ must be $t_{0}$-closed, and it must be non-trivial. We can therefore choose a gauge in which

$$
Z_{5,5} \sim \gamma_{5,2} f_{0,3}=\gamma_{5,2} A_{0,1} \stackrel{(2)}{F}_{0,2}
$$

in agreement with the previous calculation. 


\section{Invariants from superstring amplitudes}

\subsection{Heterotic superstring amplitudes}

The $N=1 d=10$ invariants constructed in the previous section can easily be verified to linearised order by comparing with heterotic superstring scattering amplitude computations using the pure spinor formalism. In comparing the invariants with scattering amplitudes, it is important to note that scattering amplitudes are computed using vertex operators constructed from superfields which satisfy linearised equations of motion. These linearised equations of motion do not receive $\alpha^{\prime}$ corrections, and are invariant under linearised supersymmetry transformations of the superfields.

However, the $N=1 d=10$ invariants of this paper are constructed using superfields which satisfy nonlinear equations of motion, and whose supersymmetry transformations are also non-linear. This means that invariants which are lowest order in $\alpha^{\prime}$ and which vanish on-shell (such as the supersymmetrisation of the Einstein-Hilbert term $\int d^{10} x \sqrt{g} R$ ) are zero (or total derivatives) using the superform method with on-shell superfields. Nevertheless, superstring scattering amplitudes will include non-linear contributions from such invariants (such as the tree-level scattering of three gravitons).

On the other hand, for invariants which are lowest order in $\alpha^{\prime}$ and do not vanish on-shell (such as the nonabelian super-Yang-Mills action), the superform method allows the construction of the complete invariant with manifest non-linear supersymmetry. However, scattering amplitudes will only be able to compute contributions from these invariants order-by-order in the linearised superfields, and these contributions will be manifestly invariant under the linearised supersymmetry.

In a flat background, heterotic superstring scattering amplitudes computed using the pure spinor formalism are expressed as

$$
\mathcal{A}=\langle f(\lambda, x, \theta)\rangle
$$

where $f(\lambda, x, \theta)=\lambda^{\alpha} \lambda^{\beta} \lambda^{\gamma} f_{\alpha \beta \gamma}(x, \theta)$ is a superfield of ghost-number 3, and \langle\rangle denotes the zero mode measure factor defined such that

$$
\left\langle\left(\lambda \gamma^{m} \theta\right)\left(\lambda \gamma^{n} \theta\right)\left(\lambda \gamma^{p} \theta\right)\left(\theta \gamma_{m n p} \theta\right)\right\rangle=1
$$

When $f(\lambda, x, \theta)$ satisfies $Q f=0$ where $Q=\lambda^{\alpha} D_{\alpha}$ is the BRST operator, the amplitude $\mathcal{A}=\langle f(\lambda, x, \theta)\rangle$ is spacetime supersymmetric. It is not difficult to verify that the resulting invariant corresponds to $J_{5,5}=\gamma_{5,2} f_{0,3}$ where $f_{0,3}=f_{\alpha \beta \gamma}$. So the superform method for constructing invariants is directly related to the zero-mode measure factor in the pure spinor formalism.

For three-point heterotic massless tree amplitudes,

$$
\mathcal{A}=\left(\alpha^{\prime}\right)^{-2}\left\langle V_{1}\left(z_{1}\right) V_{2}\left(z_{2}\right) V_{3}\left(z_{3}\right)\right\rangle
$$

where $V=\bar{c} \lambda^{\alpha}\left[A_{\alpha I}(x, \theta) J^{I}+B_{\alpha m}(x, \theta) \bar{\partial} x^{m}\right]$ is the massless vertex operator, $\bar{c}$ is the right-moving reparameterization ghost, $J^{I}$ are the right-moving currents for the gauge group, and $A_{\alpha I}$ and $B_{\alpha m}$ are the linearised superfields for super-Yang-Mills and supergravity. When $A_{\alpha I}$ and $B_{\alpha m}$ satisfy the equations of motion

$$
\left(\gamma^{m n p q r}\right)^{\alpha \beta} D_{\alpha} A_{\beta I}=\left(\gamma^{m n p q r}\right)^{\alpha \beta} D_{\alpha} B_{\beta m}=0, \quad \partial^{m} B_{\beta m}=\partial^{n} \partial_{n} A_{\beta I}=\partial^{n} \partial_{n} B_{\beta m}=0,
$$

the vertex operator $V$ is BRST-closed with respect to the left and right-moving BRST operators, $Q=$ $\int d z \lambda^{\alpha} d_{\alpha}$ and $\bar{Q}=\int d \bar{z}(\bar{c} \bar{T}+b \bar{c} \bar{\partial} c)$ where $\bar{T}$ is the right-moving stress tensor. It is easy to check that these equations for $A_{\alpha I}$ and $B_{\alpha m}$ describe the linearised on-shell super-Yang-Mills and supergravity fields. 
After integration over the right-moving worldsheet fields, one obtains the expression $\mathcal{A}=\langle f(\lambda, x, \theta)\rangle$ where

$$
\begin{aligned}
f_{\alpha \beta \gamma}= & \alpha^{\prime} \operatorname{Tr}\left(A_{\alpha}^{1} A_{\beta}^{2} A_{\gamma}^{3}\right)+\alpha^{\prime}\left(k^{2} \cdot B_{\alpha}^{1}\right)\left(k^{3} \cdot B_{\beta}^{2}\right)\left(k^{1} \cdot B_{\gamma}^{3}\right) \\
& +\alpha^{\prime}\left(B_{\alpha}^{1} \cdot k^{2}\right) \operatorname{Tr}\left(A_{\beta}^{2} A_{\gamma}^{3}\right)+\alpha^{\prime}\left(B_{\alpha}^{2} \cdot k^{3}\right) \operatorname{Tr}\left(A_{\beta}^{3} A_{\gamma}^{1}\right)+\alpha^{\prime}\left(B_{\alpha}^{3} \cdot k^{1}\right) \operatorname{Tr}\left(A_{\beta}^{3} A_{\gamma}^{1}\right) \\
& +\left(B_{\alpha}^{1} \cdot B_{\beta}^{2}\right)\left(k^{1} \cdot B_{\gamma}^{3}\right)+\left(B_{\alpha}^{2} \cdot B_{\beta}^{3}\right)\left(k^{2} \cdot B_{\gamma}^{1}\right)+\left(B_{\alpha}^{3} \cdot B_{\beta}^{1}\right)\left(k^{3} \cdot B_{\gamma}^{2}\right) .
\end{aligned}
$$

The terms in $f_{\alpha \beta \gamma}$ proportional to $\alpha^{\prime}$ correspond to the cubic on-shell terms in $W^{(2)}$ of equation (4.15), whereas the terms independent of $\alpha^{\prime}$ come from the cubic terms in the Einstein-Hilbert action. For example, $\alpha^{\prime} \operatorname{Tr}\left(A_{\alpha}^{1} A_{\beta}^{2} A_{\gamma}^{3}\right)$ is the onshell contribution to the Yang-Mills Chern-Simons term $Y_{0,3}$ in equation (4.18).

Since three-point massless amplitudes do not receive $\alpha^{\prime}$ corrections, one needs to consider higher-point amplitudes to check invariants which are higher orders in $\alpha^{\prime}$. For example, the four-point one-loop amplitude has been shown to lowest order in $\alpha^{\prime}$ to be proportional to [70] $\mathcal{A}=\left\langle\lambda^{\alpha} \lambda^{\beta} \lambda^{\gamma} f_{\alpha \beta \gamma}(x, \theta)\right\rangle$ where

$$
f_{\alpha \beta \gamma}=\operatorname{Tr}\left[A_{\alpha}^{1}\left(\gamma^{m n p q r}\right)_{\beta \gamma}\left(\Lambda^{2} \gamma_{m n p} \Lambda^{3}\right) F_{q r}^{4}\right]+\text { permutations }
$$

Comparing with (4.13), one sees that $J_{5,5}=\gamma_{5,2} f_{0,3}$ where $f_{0,3}=\operatorname{Tr}\left(A_{\alpha} F_{\beta \gamma}^{(2)}\right)$ as desired.

\section{2 $\quad N=2 d=10$ invariants}

Just as $N=1 d=10$ invariants are related to scattering amplitudes for heterotic or open superstrings, $N=2 d=10$ invariants are related to scattering amplitudes of Type II superstrings. Since vertex operators of Type II superstrings can be expressed as left-right products of open superstring vertex operators, it is natural to propose that $N=2 d=10$ invariants are related to superforms

$$
f_{0,3,3} \sim f_{\alpha \beta \gamma \hat{\alpha} \hat{\beta} \hat{\gamma}}
$$

where the first subscript denotes the number of vector indices, the second subscript denotes the number of unhatted "left-moving" spinor indices, and the third subscript denotes the number of hatted "rightmoving" spinor indices. For the Type IIA superstring, hatted and unhatted spinor indices have opposite chirality, while for the Type IIB superstring, they have the same chirality.

Type II superstring amplitudes using the pure spinor formalism are expressed as

$$
\mathcal{A}=\langle f\rangle
$$

where $f=\lambda^{\alpha} \lambda^{\beta} \lambda^{\gamma} \hat{\lambda}^{\hat{\alpha}} \hat{\lambda}^{\hat{\beta}} \hat{\lambda} \hat{\gamma} f_{\alpha \beta \gamma \hat{\alpha} \hat{\beta} \hat{\gamma}}$ is BRST closed with respect to $Q=\int d z \lambda^{\alpha} d_{\alpha}$ and $\bar{Q}=\int d \bar{z} \hat{\lambda}^{\hat{\alpha}} \hat{d}_{\hat{\alpha}}, \lambda^{\alpha}$ and $\hat{\lambda}^{\hat{\alpha}}$ are pure spinors satisfying $\lambda \gamma^{a} \lambda=\hat{\lambda} \gamma^{\alpha} \hat{\lambda}=0$, and

$$
\left\langle\left(\lambda \gamma^{m} \theta\right)\left(\lambda \gamma^{n} \theta\right)\left(\lambda \gamma^{p} \theta\right)\left(\theta \gamma_{m n p} \theta\right)\left(\hat{\lambda} \gamma^{q} \hat{\theta}\right)\left(\hat{\lambda} \gamma^{r} \hat{\theta}\right)\left(\hat{\lambda} \gamma^{s} \hat{\theta}\right)\left(\hat{\theta} \gamma_{q r s} \hat{\theta}\right)\right\rangle=1
$$

So, at least to linearised level, there is a supersymmetric $N=2 d=10$ invariant associated with $f_{0,3,3}$. For the $N=2 A$ case, one can relate this invariant with the superform method by defining

$$
\left.J_{0,5,5}=\gamma_{(\delta \epsilon}^{a b c d e} f_{\alpha \beta \gamma)(\hat{\alpha} \hat{\beta} \hat{\gamma}}\left(\gamma_{a b c d e}\right)_{\hat{\delta} \hat{\epsilon}}\right)
$$


which is the component of lowest dimension of a closed 10-form as we shall show in subsection (5.4). However, an analogous construction does not work for the $N=2 B$ case since $\gamma_{\delta \kappa}^{a b c d e}\left(\gamma_{a b c d e}\right)_{\hat{\delta} \hat{\kappa}}=0$ if the hatted and unhatted spinor indices have the same chirality.

An alternative method for constructing $N=2 A d=10$ invariants is to dimensionally reduce $N=1 d=11$ invariants. Using the pure spinor version of the $d=11$ supermembrane, amplitudes are computed as $\mathcal{A}=\langle f\rangle$ where $f$ has ghost-number seven and the zero model measure factor is of the form $\left\langle(\theta)^{9}(\lambda)^{7}\right\rangle=1$. The resulting $d=11$ invariant is obtained from the superform whose component of lowest dimension is $J_{2,9}=\gamma_{2,2} f_{0,7}$. This invariant can be obtained directly as a CS invariant starting from the closed twelveform $W=G_{4} X_{8}$, where $G_{4}$ is the supergravity four-form field strength and $X_{8}$ the anomaly-cancelling $R^{4}$ eight-form [17]. It is reasonable to assume that the lowest non-vanishing component of $K$ is $K_{3,8}$, so that $J_{2,9}=-Z_{2,9}$. From this we conclude that it is possible to take $f_{0,7}$ to be proportional to $Y_{0,7}$ where $d Y_{7}=X_{8}$.

To dimensionally reduce, suppose that the $d=11$ superform $J_{n+1,10-n}=J_{a_{1} \ldots a_{n+1}} \alpha_{1 \ldots \alpha_{10-n}}$ is independent of the $d=11$ coordinate $x^{11}$. Then it is easy to show that

$$
J_{n, 10-n}^{d=10} \equiv J_{11}^{d=11} a_{1} \ldots a_{n} \quad \alpha_{1} \ldots \alpha_{10-n}
$$

is a closed 10-form which defines an $N=2 A d=10$ invariant. Note that $J_{n, 11-n}^{d=11}$ vanishes for $n<2$, so $J_{n, 10-n}^{d=10}$ vanishes for $n<1$ when defined using this method.

An obvious question is how to relate these superstring and supermembrane constructions of $N=2 A d=$ 10 invariants. The relation is not obvious since the supermembrane method constructs an invariant whose component of lowest dimension is $J_{1,9}$, whereas the invariant constructed using the superstring method has $J_{0,10}$ as its component of lowest dimension. Furthermore, $J_{0,10}$ constructed using the superstring method has five hatted and five unhatted indices, whereas the supermembrane-derived $J_{1,9}$ naively has no restriction on the relative number of hatted and unhatted spinor indices.

\subsection{Proposal for $N=2$ invariants using doubled superspace}

In the previous subsection, we presented two approaches for constructing $N=2 A$ invariants using the superform method. However, despite the fact that Type IIA and Type IIB superstrings are related by T-duality, neither of these approaches appear to be useful for constructing $N=2 B$ invariants. In this subsection, we present an alternative proposal for constructing $N=2$ invariants which works equally well for $N=2 A$ and $N=2 B$. However, we have not yet checked the consistency of this proposal at the non-linear level.

This alternative proposal for constructing $N=2$ supersymmetric invariants involves doubling both the number of bosonic coordinates and fermionic coordinates; it was first discussed in [71, 72]. The bosonic coordinates in this "doubled" superspace will be called $x^{m}$ and $\hat{x}^{\hat{m}}$ for $m, \hat{m}=0$ to 9 , and the fermionic coordinates will be called $\theta^{\mu}$ and $\hat{\theta}^{\hat{\mu}}$ for $\mu, \hat{\mu}=1$ to 16 . One then defines the supervierbeins $E_{M}{ }^{A}$ and $E_{\hat{M}} \hat{A}$ where $M=(m, \mu)$ and $\hat{M}=(\hat{m}, \hat{\mu})$ describe the curved coordinates and $A=(a, \alpha)$ and $\hat{A}=(\hat{a}, \hat{\alpha})$ describe the tangent-space indices. In a flat background, the only non-vanishing torsions will be defined to be $T_{\alpha \beta}{ }^{a}=-i\left(\gamma^{a}\right)_{\alpha \beta}$ and $T_{\hat{\alpha} \hat{\beta}}^{\hat{a}}=-i\left(\gamma^{\hat{a}}\right)_{\hat{\alpha} \hat{\beta}}$.

Such a doubled formalism has previously appeared in discussions of T-duality for the heterotic superstring $[73,74]$ as well as in the Type II superstring. Although we have not yet checked the consistency of the latter construction at the non-linear level (see [75] for some partical results on this), we think it is a promising possibility for constructing $N=2 d=10$ invariants using the superform method.

Superforms $f_{A_{1} \ldots A_{10} ; \hat{A}_{1} \ldots \hat{A}_{10}}$ in this "doubled" superspace contain 10 unhatted indices and 10 hatted indices, where $A$ is either $a$ or $\alpha$ and $\hat{A}$ is either $\hat{a}$ or $\hat{\alpha}$. Furthermore, all components $f$ of this superform are constrained to satisfy 


$$
\frac{\partial}{\partial x^{m}} f=\frac{\partial}{\partial \hat{x}^{\hat{m}}} f
$$

so that $f$ only depends on 10 bosonic and 32 fermionic coordinates. It is easy to see that if $f$ is a closed 20 -form satisfying the above constraint the integral

$$
\left.\int d^{10} x\right|_{\hat{x}=x} \epsilon^{m_{1} \ldots m_{10}} \epsilon^{\hat{m}_{1} \ldots \hat{m}_{10}} f_{m_{1} \ldots m_{10} ; \hat{m}_{1} \ldots \hat{m}_{10}}
$$

is invariant under $N=2 d=10$ supersymmetry where $\int d^{10} x_{\hat{x}=x}$ means that one is to set $\hat{x}^{\hat{m}}=x^{m}$ and integrate over $x^{m}$. Note that this invariant can also be written as

$$
\int d^{10} x \int d^{10} \hat{x} \epsilon^{m_{1} \ldots m_{10}} \epsilon^{\hat{m}_{1} \ldots \hat{m}_{10}} f_{m_{1} \ldots m_{10} ; \hat{m}_{1} \ldots \hat{m}_{10}}
$$

where $x^{m}-\hat{x}^{\hat{m}}$ is defined to take values on a compact space of unit volume.

Using this doubled superspace, we conjecture that a closed $(10 ; 10)$ superform can be constructed such that the component of $f$ with lowest dimension is

$$
f_{5,5 ; 5,5}=\gamma_{5,2 ; 0,0} \gamma_{0,0 ; 5,2} f_{0,3 ; 0,3}
$$

where $\gamma_{5,2 ; 0,0}=\gamma_{\alpha \beta}^{m_{1} \ldots m_{5}}, \gamma_{0,0 ; 5,2}=\gamma_{\hat{\alpha} \hat{\beta}}^{\hat{m}_{1} \ldots \hat{m}_{5}}$, and $f_{0,3 ; 0,3}$ is defined in Eq. (5.7). At the linearised level, it is easy to see that such a definition would lead to the invariants described by Type II superstring amplitudes using the measure factor of Eq. (5.9). For example, just as the cubic super-Yang-Mills amplitude is described by $f_{0,3}=A_{\alpha} A_{\beta} A_{\gamma}$ where $A_{\alpha}$ is the spinor super-Yang-Mills gauge potential, the cubic contribution to $N=2$ supergravity is described by $f_{0,3 ; 0,3}=B_{\alpha \hat{\alpha}} B_{\beta \hat{\beta}} B_{\gamma \hat{\gamma}}$ where $B_{\alpha \hat{\alpha}}$ is the spinorspinor component of the antisymmetric tensor superfield which can be interpreted as the "left-right" product of two spinor super-Yang-Mills gauge potentials.

\subsection{Remarks on $N=2, d=10$ cohomology}

Provided that the $H_{t}^{p, q}$ cohomology groups are determined by scalar branes, for $N=2, d=10$ it follows that these will only be non-zero for $p=0,1$, since there are only strings in this case. Consider a $t_{0}$-closed $(1, q)$-form $\omega$ in IIA; it can be written

$$
\omega_{1, q}=\Gamma_{1,2}^{\natural} \mu_{0, q-2},
$$

where $\Gamma_{1,2}^{\natural}$ denotes $\Gamma_{a} \Gamma_{11}$ viewed as a $(1,2)$-form and where $\Gamma$ denotes the $32 \times 32$ Dirac matrices. Now $\omega$ will change by a $t_{0}$ exact term if $\mu$ does, but it will also do so if $\mu$ is changed by $\Gamma_{0,2}^{\natural} \rho_{0, q-4}$. This means that the $d=10$ IIA group $H_{t}^{1, q}$ is isomorphic to the $d=11$ group $H_{t}^{0, q-2}, q \geq 2$. This explains how dimensional reduction from $d=11$ to $N=2, d=10$ works cohomologically because the relevant groups are still the $d=11$ ones.

In IIB there are two strings and hence two possible $(1,2)$-forms which can be used to write down elements of $H_{t}^{1, q}$.

There are other groups we can consider in $N=2$ as there is now a triple-grading of forms: $\Omega_{p, q}=$ $\sum_{r+s=q} \Omega_{p, r, s}$, where the $(r, s)$ labels correspond to unhatted and hatted odd indices. We also have

$$
t_{0}=\tau_{0}+\hat{\tau}_{0}
$$




$$
\begin{aligned}
& d_{1}=\partial_{1}+\hat{\partial}_{1} \\
& t_{1}=\tau_{1}+\hat{\tau}_{1},
\end{aligned}
$$

where the tri-degrees are $(-1,2,0)\left((-1,0,2)\right.$ for $\tau_{0}\left(\hat{\tau}_{0}\right),(0,1,0)\left((0,0,1)\right.$ for $\partial_{1}\left(\hat{\partial}_{1}\right)$ and $(2,-1,0)$ $((2,0,-1))$ for $\tau_{1}\left(\hat{\tau}_{1}\right)$. In principle one could also have a component of $t_{0}$ with tri-degree $(-1,1,1)$ but it vanishes in on-shell supergravity. One can easily write out $d^{2}=0$ in terms of these operations. There are various cohomology groups that can be constructed. For example, one can define $H_{\tau}^{p, r, s}$, the space of $\tau_{0}$-closed $(p, r, s)$-forms modulo the exact ones. Since $\partial_{1} \tau_{0}+\tau_{0} \partial_{1}=0$ and $\partial_{1}^{2}+\tau_{0} d_{0}+d_{0} \tau_{0}=0$ we can define spinorial cohomology groups of the form $H_{s}^{p, r, s}$, and similarly for the hatted sector. However, in general it does not seem to be very easy to analyse the spinorial cohomology groups we are interested in terms of these partial ones.

We shall give one example, in IIA, which relates to the $J_{0,5,5}$ discussed in Eq.(5.10). Suppose we have a $(0, r, s)$-form $\omega_{0, r, s}$, with $r, s \geq 3$, and suppose that

$$
\omega_{0, r, s}=\Gamma_{0,2,2} f_{0, r-2, s-2}
$$

where

$$
\Gamma_{0,2,2}:=\gamma_{5,2,0} \gamma^{5}, 0,2
$$

i.e. the contraction of two five-index gamma-matrices considered as a $(0,2,2)$-form. We would like to construct a closed $(r+s)$-form $\omega$ starting from $\omega_{0, r, s}$. We can solve the first non-trivial component of $d \omega=0$ if

$$
\begin{aligned}
\partial_{1} \omega_{0, r, s}+\tau_{0} \omega_{1, r-1, s} & =0 \\
\hat{\tau}_{0} \omega_{1, r-1, s} & =0,
\end{aligned}
$$

and similarly for the hatted components. The first of these is satisfied if

$$
\partial_{1} f_{0, r-2, s-2}+\tau_{0} f_{1, r-3, s-2}=0 .
$$

If this is so, then

$$
\begin{aligned}
\partial_{1} \omega_{0, r, s} & =-\Gamma_{0,2,2} \tau_{0} f_{1, r-3, s-2} \\
& =-\left(\gamma_{5,2,0} \gamma^{5}{ }_{, 0,2}\right)\left(\gamma_{1,2,0} f^{1}{ }_{, r-3, s-2}\right) .
\end{aligned}
$$

Now we can use the fact that $\gamma_{5,2,0} \gamma_{1,2,0}=0$ to shuffle the indices so that the even index on $\gamma_{1,2,0}$ is contracted with one of the even indices of $\gamma_{, 0,2}^{5}$. This means that we can choose $\omega_{1, r-1, s}$ to be

$$
\omega_{1, r-1, s}=\gamma_{5,0,2} g^{4}, r-1, s-2
$$

where

$$
g_{4, r-1, s-2} \sim \gamma_{5,2,0} f^{1}{ }_{, r-3, s-2} .
$$

It is now immediate that $\omega_{1, r-1, s}$ defined by $(5.23)$ is annihilated by $\hat{\tau}_{0}$. Similar considerations apply to $\omega_{1, r, s-1}$. 


\section{Discussion}

In this paper, we combined the superform method with pure spinor cohomology to construct invariants with manifest $N=1 d=10$ supersymmetry. This method was used to construct on-shell invariants of the heterotic superstring effective action up to order $\left(\alpha^{\prime}\right)^{3}$ corrections including supersymmetrisation of the $F^{2}, R^{2}, F^{4}, R^{4}, B F^{4}$ and $B R^{4}$ terms. Although we did not attempt to expand these invariants in terms of component fields it should be straightforward to perform this component expansion by evaluating the $J_{n, 10-n}$ forms which appear in the invariants.

There are several possible generalisations of our results. One obvious one is to construct invariants for terms in the effective action which are higher-order in $\alpha^{\prime}$. It would be very interesting to identify restrictions imposed by supersymmetry which constrain the possible couplings to the dilaton. Since the dilaton counts loops, these restrictions might be used for testing string duality conjectures as in [76].

Another possible generalisation is to use the superform method to construct $d=11$ and $N=2 d=10$ invariants. As discussed in section (5.2), there are some unresolved puzzles concerning the relation of these invariants, and it would also be of interest to investigate further the doubled superspace proposal of section (5.3).

Acknowledgements: We would like to thank Carlos Mafra for useful discussions and the Isaac Newton Institute for Mathematical Sciences for their hospitality during the workshop on Strong Fields, Integrability and Strings. NB would like to thank CNPq grant 305814/2006-0 and FAPESP grant 04/11426-0 for partial financial support. This work was also supported in part by EU grant (superstring theory) MRTN-2004-512194.

\section{References}

[1] B. E. W. Nilsson, "Pure Spinors As Auxiliary Fields In The Ten-Dimensional Supersymmetric YangMills Theory," Class. Quant. Grav. 3 (1986) L41.

[2] P. S. Howe, "Pure Spinors Lines In Superspace And Ten-Dimensional Supersymmetric Theories," Phys. Lett. B 258 (1991) 141 [Addendum-ibid. B 259 (1991) 511].

[3] P. S. Howe, "Pure Spinors, Function Superspaces And Supergravity Theories In Ten-Dimensions And Eleven-Dimensions," Phys. Lett. B 273 (1991) 90.

[4] M. Tonin, "World sheet supersymmetric formulations of Green-Schwarz superstrings," Phys. Lett. B 266 (1991) 312.

[5] N. Berkovits, "The heterotic Green-Schwarz superstring on an $N=(2,0)$ superworldsheet," Nucl. Phys. B 379 (1992) 96 [arXiv:hep-th/9201004].

[6] M. Matone, L. Mazzucato, I. Oda, D. Sorokin and M. Tonin, "The superembedding origin of the Berkovits pure spinor covariant quantization of superstrings," Nucl. Phys. B 639 (2002) 182 [arXiv:hep-th/0206104].

[7] N. Berkovits, "Super-Poincare covariant quantization of the superstring," JHEP 0004 (2000) 018 [arXiv:hep-th/0001035].

[8] N. Berkovits, "ICTP lectures on covariant quantization of the superstring," arXiv:hep-th/0209059.

[9] N. Berkovits, "Towards covariant quantization of the supermembrane," JHEP 0209 (2002) 051 [arXiv:hep-th/0201151].

[10] P. A. Grassi and P. Vanhove, "Topological M theory from pure spinor formalism," Adv. Theor. Math. Phys. 9 (2005) 285 [arXiv:hep-th/0411167]. 
[11] L. Anguelova, P. A. Grassi and P. Vanhove, "Covariant one-loop amplitudes in $D=11$," Nucl. Phys. B702 (2004) 269 [arXiv:hep-th/0408171].

[12] P. Fre' and P. A. Grassi, "Pure spinors, free differential algebras, and the supermembrane," Nucl. Phys. B 763 (2007) 1 [arXiv:hep-th/0606171].

[13] P. Fre and P. A. Grassi, "Free Differential Algebras, Rheonomy, and Pure Spinors," arXiv:0801.3076 [hep-th].

[14] R. D'Auria, P. Fre', P. A. Grassi and M. Trigiante, "Pure Spinor Superstrings on Generic type IIA Supergravity Backgrounds," arXiv:0803.1703 [hep-th].

[15] P. Fre' and P. A. Grassi, "Pure Spinors for General Backgrounds," arXiv:0803.1809 [hep-th].

[16] M. Cederwall, B. E. W. Nilsson and D. Tsimpis, "Spinorial cohomology and maximally supersymmetric theories," JHEP 0202 (2002) 009 [arXiv:hep-th/0110069].

[17] P. S. Howe and D. Tsimpis, "On higher-order corrections in M theory," JHEP 0309 (2003) 038 [arXiv:hep-th/0305129].

[18] M. Cederwall, B. E. W. Nilsson and D. Tsimpis, "The structure of maximally supersymmetric Yang-Mills theory: Constraining higher-order corrections," JHEP 0106 (2001) 034 [arXiv:hepth/0102009].

[19] M. Cederwall, B. E. W. Nilsson and D. Tsimpis, "D = 10 super-Yang-Mills at O(alpha**2)," JHEP 0107 (2001) 042 [arXiv:hep-th/0104236].

[20] M. Cederwall, B. E. W. Nilsson and D. Tsimpis, "Spinorial cohomology of abelian d $=10$ superYang-Mills at O(alpha'**3)," JHEP 0211 (2002) 023 [arXiv:hep-th/0205165].

[21] P. S. Howe, S. F. Kerstan, U. Lindstrom and D. Tsimpis, "The deformed M2-brane," JHEP 0309 (2003) 013 [arXiv:hep-th/0307072].

[22] J. M. Drummond and S. F. Kerstan, "Kappa-symmetric derivative corrections to D-brane dynamics," JHEP 0410 (2004) 006 [arXiv:hep-th/0407145].

[23] J. M. Drummond and S. F. Kerstan, "Kappa-symmetric deformations of M5-brane dynamics," JHEP 0506 (2005) 003 [arXiv:hep-th/0412149].

[24] M. Cederwall and B. E. W. Nilsson, "Pure Spinors and D=6 Super-Yang-Mills," arXiv:0801.1428 [hep-th].

[25] S. J. J. Gates, "Ectoplasm has no topology: The prelude," arXiv:hep-th/9709104.

[26] S. J. J. Gates, M. T. Grisaru, M. E. Knutt-Wehlau and W. Siegel, "Component actions from curved superspace: Normal coordinates and ectoplasm," Phys. Lett. B 421 (1998) 203 [arXiv:hepth/9711151].

[27] S. J. J. Gates, "Ectoplasm has no topology," Nucl. Phys. B 541 (1999) 615 [arXiv:hep-th/9809056].

[28] P.S. Howe, "Pure spinors and spinorial cohomology." Talk given at the IHES/CEA Workshop on the Pure Spinor Formalism in String Theory, Bures-sur-Yvette, France, January 2005.

[29] N. Berkovits, "Explaining pure spinor superspace," arXiv:hep-th/0612021.

[30] R. D'Auria, P. Fre, P. K. Townsend and P. van Nieuwenhuizen, "Invariance Of Actions, Rheonomy And The New Minimal N=1 Supergravity In The Group Manifold Approach," Annals Phys. 155 (1984) 423.

[31] I. A. Bandos, D. P. Sorokin and D. Volkov, "On the generalized action principle for superstrings and supermembranes," Phys. Lett. B 352 (1995) 269 [arXiv:hep-th/9502141]. 
[32] L. Bonora, P. Pasti and M. Tonin, "Superspace Formulation Of 10-D Sugra+Sym Theory A La Green-Schwarz," Phys. Lett. B 188 (1987) 335.

[33] P. S. Howe, K. S. Stelle and P. K. Townsend, "Superactions," Nucl. Phys. B 191 (1981) 445.

[34] A. Galperin, E. Ivanov, S. Kalitsyn, V. Ogievetsky and E. Sokatchev, "Unconstrained N=2 Matter, Yang-Mills And Supergravity Theories In Harmonic Superspace," Class. Quant. Grav. 1 (1984) 469.

[35] G. G. Hartwell and P. S. Howe, "(N, P, Q) Harmonic Superspace,” Int. J. Mod. Phys. A 10 (1995) 3901 [arXiv:hep-th/9412147].

[36] T. Biswas and W. Siegel, "N = 2 harmonic superforms, multiplets and actions," JHEP 0111 (2001) 004 [arXiv:hep-th/0105084].

[37] P. S. Howe, O. Raetzel and E. Sezgin, "On brane actions and superembeddings," JHEP 9808 (1998) 011 [arXiv:hep-th/9804051].

[38] L. Bonora, P. Pasti and M. Tonin, "Chiral anomalies in higher dimensional supersymmetric theories," Nucl. Phys. B 286 (1987) 150.

[39] N. Berkovits, "Cohomology in the pure spinor formalism for the superstring," JHEP 0009 (2000) 046 [arXiv:hep-th/0006003].

[40] B. E. W. Nilsson, "Simple Ten-Dimensional Supergravity In Superspace," Nucl. Phys. B 188 (1981) 176.

[41] P. S. Howe, H. Nicolai and A. Van Proeyen, "Auxiliary Fields And A Superspace Lagrangian For Linearized Ten-Dimensional Supergravity," Phys. Lett. B 112 (1982) 446.

[42] E. Bergshoeff and M. de Roo, "The Supercurrent In Ten-Dimensions," Phys. Lett. B 112 (1982) 53.

[43] B. E. W. Nilsson and A. K. Tollsten, "The Geometrical Off-Shell Structure Of Pure N=1 D = 10 Supergravity In Superspace," Phys. Lett. B 169 (1986) 369.

[44] P. S. Howe and A. Umerski, "On superspace supergravity in ten dimensions," Phys. Lett. B 177 (1986) 163.

[45] R. E. Kallosh and B. E. W. Nilsson, "Scale Invariant D = 10 Superspace And The Heterotic String," Phys. Lett. B 167 (1986) 46.

[46] B. E. W. Nilsson and A. K. Tollsten, "Superspace Formulation Of The Ten-Dimensional Coupled Einstein Yang-Mills System," Phys. Lett. B 171 (1986) 212.

[47] J. J. Atick, A. Dhar and B. Ratra, "Superspace Formulation Of Ten-Dimensional N=1 Supergravity Coupled To N=1 Superyang-Mills Theory," Phys. Rev. D 33 (1986) 2824.

[48] R. D'Auria, P. Fre, M. Raciti and F. Riva, "Anomaly free supergravity in $D=10$. 1. The Bianchi Identities and the bosonic Lagrangian," Int. J. Mod. Phys. A 3 (1988) 953.

[49] M. Raciti, F. Riva and D. Zanon, "Perturbative Approach To D = 10 Superspace Supergravity With A Lorentz Chern-Simons Form," Phys. Lett. B 227 (1989) 118.

[50] L. Bonora, M. Bregola, K. Lechner, P. Pasti and M. Tonin, "Anomaly free supergravity and super Yang-Mills in ten dimensions," Nucl. Phys. B 296 (1988) 877.

[51] L. Bonora, K. Lechner, M. Bregola, P. Pasti and M. Tonin, "A Discussion of the constraints in N=1 SUGRA-SYM in 10-D," Int. J. Mod. Phys. A 5 (1990) 461.

[52] L. Bonora et al., "Some remarks on the supersymmetrization of the Lorentz Chern-Simons form in $\mathrm{D}=10 \mathrm{~N}=1$ supergravity theories," Phys. Lett. B 277 (1992) 306. 
[53] S. Bellucci and S. J. J. Gates, "D = 10, N=1 Superspace supergravity and the Lorentz Chern-Simons form," Phys. Lett. B 208 (1988) 456.

[54] S. Bellucci, D. A. Depireux and S. J. J. Gates, "Consistent and universal inclusion of the Lorentz Chern-Simons form in $\mathrm{D}=10, \mathrm{~N}=1$ supergravity theories," Phys. Lett. B 238 (1990) 315.

[55] S. J. J. Gates, A. Kiss and W. Merrell, "Dynamical equations from a first-order perturbative superspace formulation of 10D, N = 1 string-corrected supergravity. I," JHEP 0412 (2004) 047 [arXiv:hepth/0409104].

[56] N. A. Saulina, M. V. Terentev and K. N. Zyablyuk, "Duality In N=1, D = 10 Superspace And Supergravity With Tree Level Superstring Corrections," Phys. Lett. B 366 (1996) 134 [arXiv:hepth/9507033].

[57] N. A. Saulina, M. V. Terentev and K. N. Zyablyuk, "Five-brane Lagrangian with loop corrections in field-theory limit," Int. J. Mod. Phys. A 12 (1997) 4559 [arXiv:hep-th/9607079].

[58] K. Lechner and M. Tonin, "Superspace formulations of ten-dimensional supergravity," arXiv:0802.3869 [hep-th].

[59] B. E. W. Nilsson, "Off-Shell D = 10, N=1 Poincare Supergravity And The Embeddibility Of Higher Derivative Field Theories In Superspace," Phys. Lett. B 175 (1986) 319.

[60] A. Candiello and K. Lechner, "The Supersymmetric version of the Green-Schwarz anomaly cancellation mechanism," Phys. Lett. B 332 (1994) 71 [arXiv:hep-th/9404095].

[61] S. J. J. Gates and S. Vashakidze, "On D = 10, N=1 Supersymmetry, superspace geometry and superstring effects," Nucl. Phys. B 291 (1987) 172.

[62] E. Bergshoeff, M. Rakowski and E. Sezgin, "Higher Derivative Superyang-Mills Theories," Phys. Lett. B 185 (1987) 371.

[63] D. J. Gross and J. H. Sloan, "The Quartic Effective Action for the Heterotic String," Nucl. Phys. B 291 (1987) 41.

[64] E. Bergshoeff and M. de Roo, "Supersymmetric Chern-Simons terms in ten-dimensions," Phys. Lett. B 218 (1989) 210.

[65] E. A. Bergshoeff and M. de Roo, "The Quartic Effective Action Of The Heterotic String And Supersymmetry," Nucl. Phys. B 328 (1989) 439.

[66] M. de Roo, H. Suelmann and A. Wiedemann, "The Supersymmetric effective action of the heterotic string in ten-dimensions," Nucl. Phys. B 405 (1993) 326 [arXiv:hep-th/9210099].

[67] B. E. W. Nilsson and A. K. Tollsten, "Supersymmetrization of zeta (3) R**4 in superstring theories," Phys. Lett. B 181 (1986) 63.

[68] K. Lechner, P. Pasti and M. Tonin, "Anomaly free Sugra and the $\mathrm{R}^{* *} 4$ superstring term," Mod. Phys. Lett. A 2, 929 (1987).

[69] K. Lechner and P. Pasti, "Nonminimal anomaly free D=10, N=1 Sugra-SYM and four graviton superstring amplitudes," Mod. Phys. Lett. A 4 (1989) 1721.

[70] N. Berkovits, "Multiloop amplitudes and vanishing theorems using the pure spinor formalism for the superstring," JHEP 0409 (2004) 047 [arXiv:hep-th/0406055].

[71] A. P. Isaev and E. A. Ivanov, "On Sigma Model Formulation Of Green-Schwarz Superstring," Mod. Phys. Lett. A 4 (1989) 351.

[72] A. P. Isaev and E. A. Ivanov, "Green-Schwarz Superstring As An Asymmetric Chiral Field Sigma Model," Theor. Math. Phys. 81 (1990) 1304 [Teor. Mat. Fiz. 81 (1989) 420]. 
[73] W. Siegel, "Two vierbein formalism for string inspired axionic gravity," Phys. Rev. D 47 (1993) 5453 [arXiv:hep-th/9302036].

[74] W. Siegel, "Superspace duality in low-energy superstrings," Phys. Rev. D 48 (1993) 2826 [arXiv:hepth/9305073].

[75] A. P. Isaev and E. A. Ivanov, "Nonabelian $\mathrm{N}=2$ superstrings," preprint IC-90-97.

[76] M. B. Green and S. Sethi, "Supersymmetry constraints on Type IIB supergravity," Phys. Rev. D 59 (1999) 046006 [arXiv:hep-th/9808061]. 